
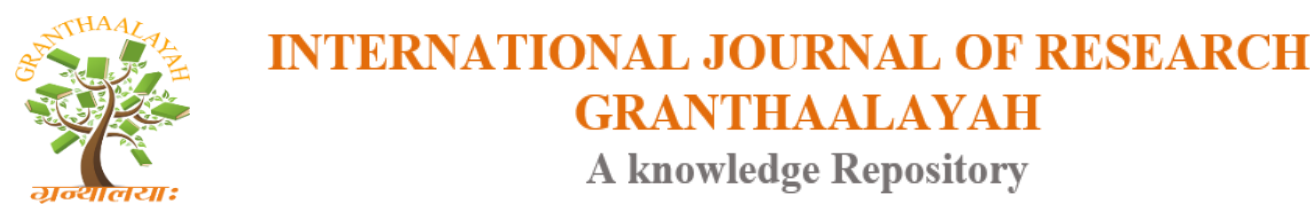

Science

\title{
SIMILAR RETINAL FINDINGS IN TRAUMATIC BRAIN INJURY AND ALZHEIMER'S
}

\author{
Selcuk N. Cekmeceli ${ }^{1}$, Umur A. Kayabasi ${ }^{2}$ \\ ${ }^{1}$ Ophthalmologist Bahcesehir University- Istanbul \\ ${ }^{2}$ Neuro-Ophthalmologist Bahcesehir University- Istanbul
}

\begin{abstract}
Aim: Our aim was to show similarities between retinal findings in Alzheimer's disease (AD) and Traumatic Brain Injury (TBI).

Methods: 30 patients with AD, 4 patients with TBI and 20 age-matched healthy controls were examined by FAF and SD-OCT to find amyloid plaques and tau tangles in the retina.

Results: Similar images of plaques and tau tangles were detected in both diseases.

Conclusion: TBI may cause neurodegeneration in the retina similar to AD.

Keywords: Similar Retinal; Traumatic; Brain Injury; Alzheimer’s

Cite This Article: Selcuk N. Cekmeceli, and Umur A. Kayabasi. (2018). "SIMILAR RETINAL FINDINGS IN TRAUMATIC BRAIN INJURY AND ALZHEIMER'S.” International Journal of Research - Granthaalayah, 6(11), 330-336. 10.29121/granthaalayah.v6.i11.2018.1135.
\end{abstract}

\section{Introduction}

Alzheimer's disease (AD), first described by German psychiatrist and neuropathologist Alois Alzheimer in 1906 is the most common form of dementia. (1) Most often, AD is diagnosed In people over 65 years of age, although the less prevalent early-onset AD can occur much EarlierAD is a chronic neurodegenerative disease characterized by loss of memory And cognitive decline and is neuropathologically associated with an increase in beta- amyloid Plaques, neurofibrillary tangles and other degenerative aggregates (2) A previous history of Traumatic brain injury (TBI) has been reported to increase the incidence of AD and other dementias. (3) Repeated mild TBI in boxers, American football players and military personnel have been linked With arly neuropathological changes in the brain (4) the deposition of hyperphosphorylated Tau Has been proposed as the pathognomonic feauture of TBI which is also the culprit in AD that is a Process beginning many years before symptoms of dementia becomes evident. (3) New research Has found changes in the brain and body up to 20 years before $\mathrm{AD}$ symptoms arise. $(5,6)$

\section{Lipofuscin Hypothesis of AD}

Lipofuscin is probably one of the most studied pigments of aging. As people age, it starts to accumulate in many tissues .(7) There is much evidence that lipofuscin is an important In aging 
and neurodegenerative diseases It is an end result of free radical damage to biological membranes. Cell membranes are damaged by oxygen free radicals through lipid Peroxidation and the molecular garbage formed turns into lipofuscin. (8)

Lipofuscin may give damage to the tissues by mechanically obstructing the flow into and out of the cells and slowing down the elimination of waste materials. The other waste pigments are ceroid and amyloid a form of which is beta amyloid and it plays a major role in AD. (8)

\section{Lipofuscin in the Retina}

In the retinal pigment epithelium (RPE), lipofuscin is formed as a by-product of phagocytosis of photoreceptors over age 70, lipofuscin may occupy more than $20 \%$ of the cytoplasmic space of The cell Excessive levels of lipofuscin may compromise RPE and contribute to age-related maculardegeneration (AMD). $(7,8)$ Fundus autofluorescein (FAF) detects lipofuscin in the retina (9)

\section{Lipofuscin, Beta-Amyloid Plaques and Tau Tangles}

In a study, a high-resolution, noninvasive optical imaging device was used to identify beta-amyloid Plaques in the retinas of live mice. (10) The research team used a fluorescent compound (curcumin) to detect retinal plaques. Characteristic amyloid plaques in retinas of $\mathrm{AD}$ patients and live laboratory mice were shown with this noninvasive optical imaging technique. Beta-amyloid plaques which damage cells were recognized as the hallmark sign of AD. $(10,11)$ Tau tangles are the other toxic proteins which cause neuronal death and may be found in close contact with alphasynuclein. Other than amyloid beta, according to a very recent study Tauprotein is the culprit in $\mathrm{AD}$ and the major damage to the neurons stems from the accumulation of toxic Tau .(12)

\section{Materials and Methods}

4 patients with a history of severe TBI due to motor vehicle accident were examined by FAF and SD-OCT. All the patients were seen after 6 months of the initial event. Their neurological rehabilitation was performed at other centers and they were referred to our hospital for retina tests.

Cranial MRI images disclosed hippocampal atrophy in all of them. (Figure 1) Age range was 51 (28-69). Hypo or hyperfluorescent regions were detected by FAF and SD-OCT was performed through these lesions to find the layer of accumulations. Curcumin capsules $(80 \mathrm{mg}$ bid for three days) were given to all the patients. First, the areas detected by FAF were observed with SD-OCT, and then most parts of the retina were inspected. Plaque, Tau tangle and alpha-synuclein images were compared with the histopathologic imagings. (Figure 2) 


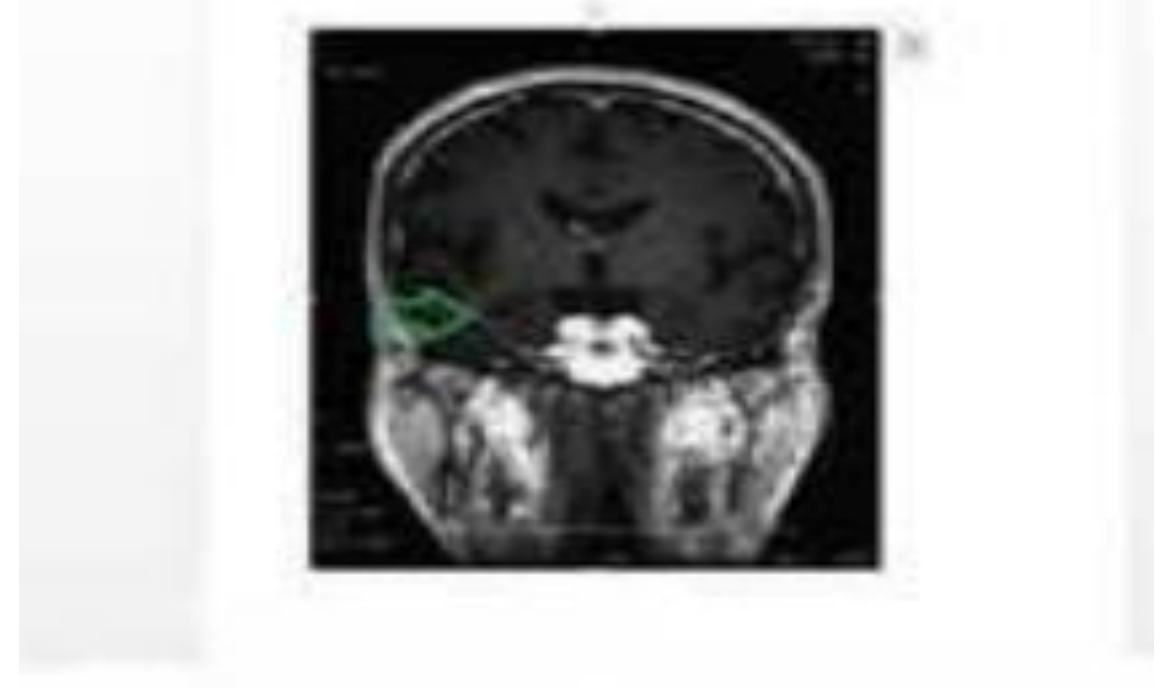

Figure 1: Hippocampal atrophy on MRI.

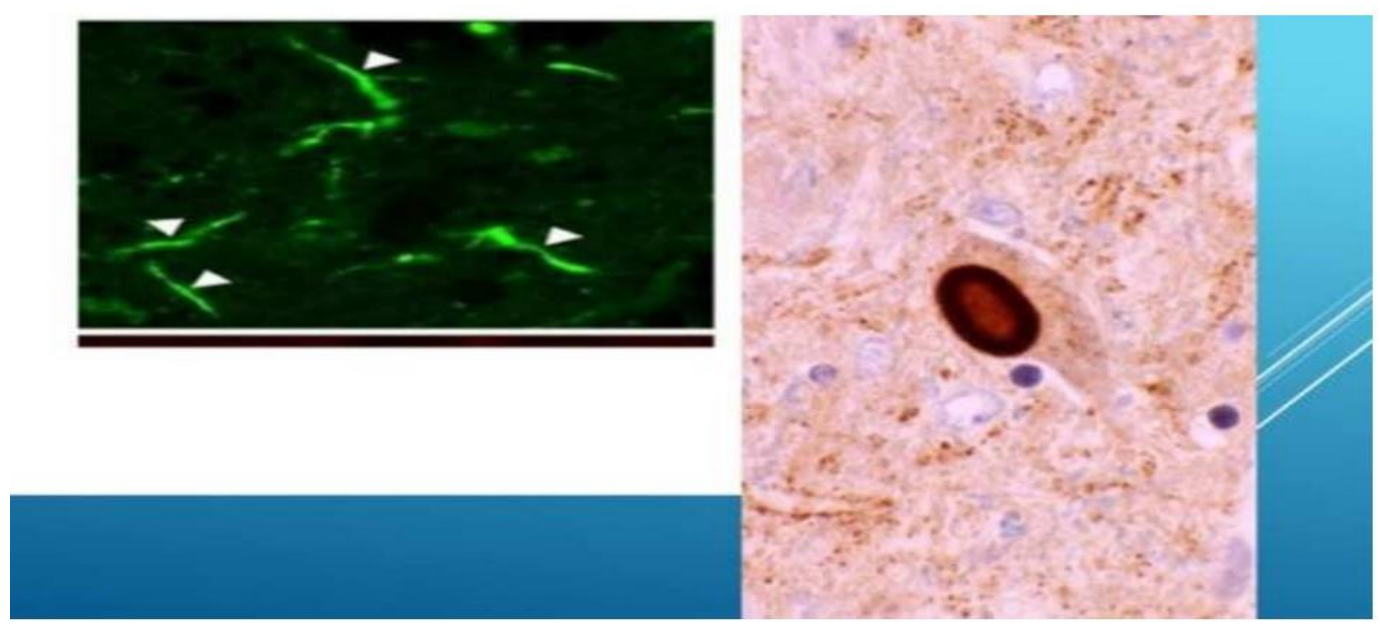

Figure 2: Histopathologic images of Tau and alpha-synuclein.

Identical images were taken into consideration. Abnormal protein aggregations in the retina were detected by two observers in a masked fashion. The observers agreed on the final appearance of the lesions.

\section{Results}

In all the patients Tau tangles and curcumin stained plaques were detected. Tau tangles and plaques were most frequently found in hypo or hyperfluorescent regions detected by FAF. The stained plaques were commonly found in the outer plexiform and ganglion layers. Tau tangles occupied many layers and extended to the ganglion layer. (Figures 3, 4)Tau tangles were found either isolated or in close contact with alpha-synuclein. (Figure 5) 


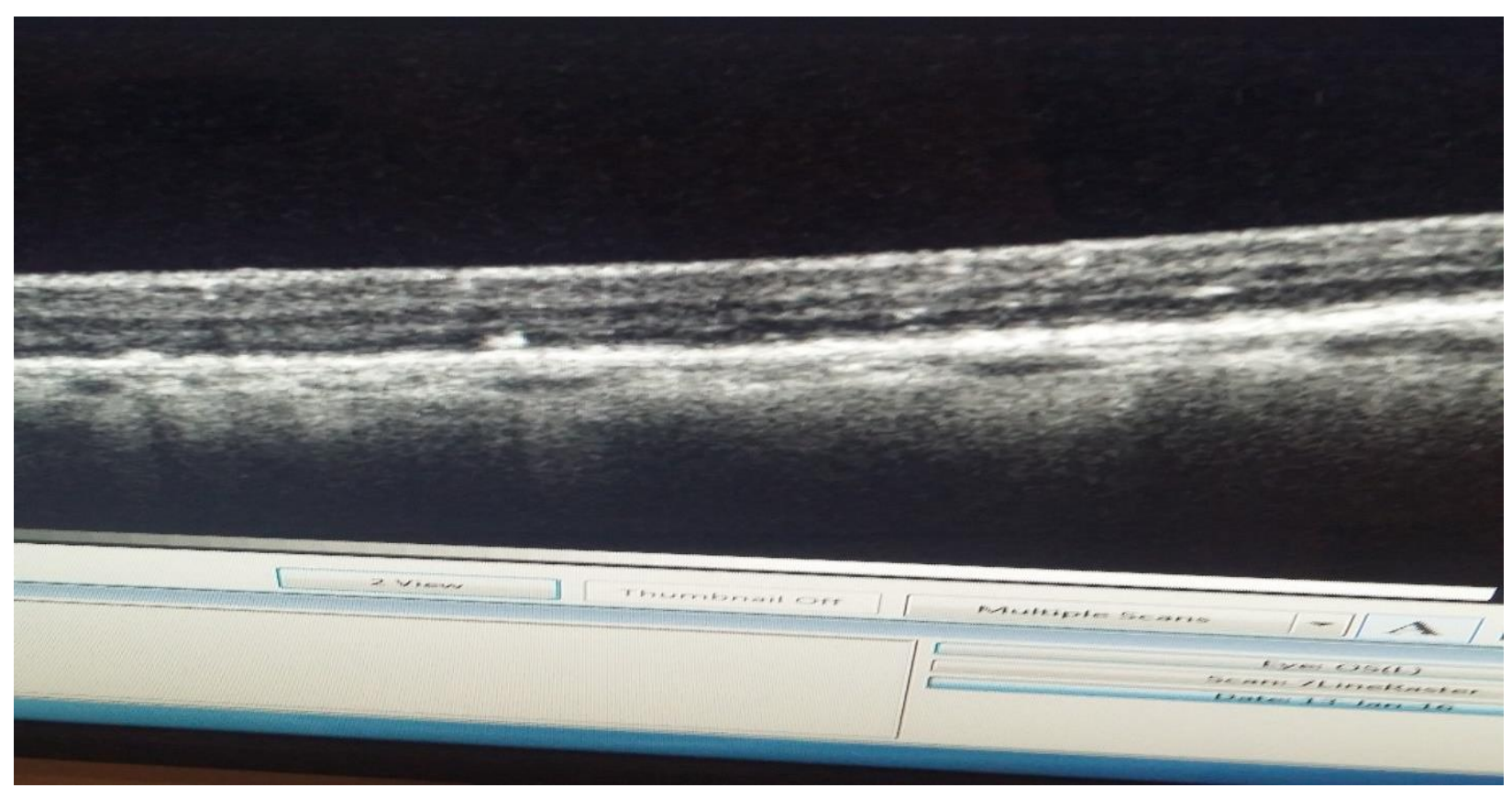

Figure 3: Beta amyloid plaques.

The appearance of the abnormal protein accumulations looked very similar to the neurodegenerative changes in $\mathrm{AD}$. Curcumin stained plaques were mostly located in the temporal retina, again identical to the plaque localizations in $\mathrm{AD}$.

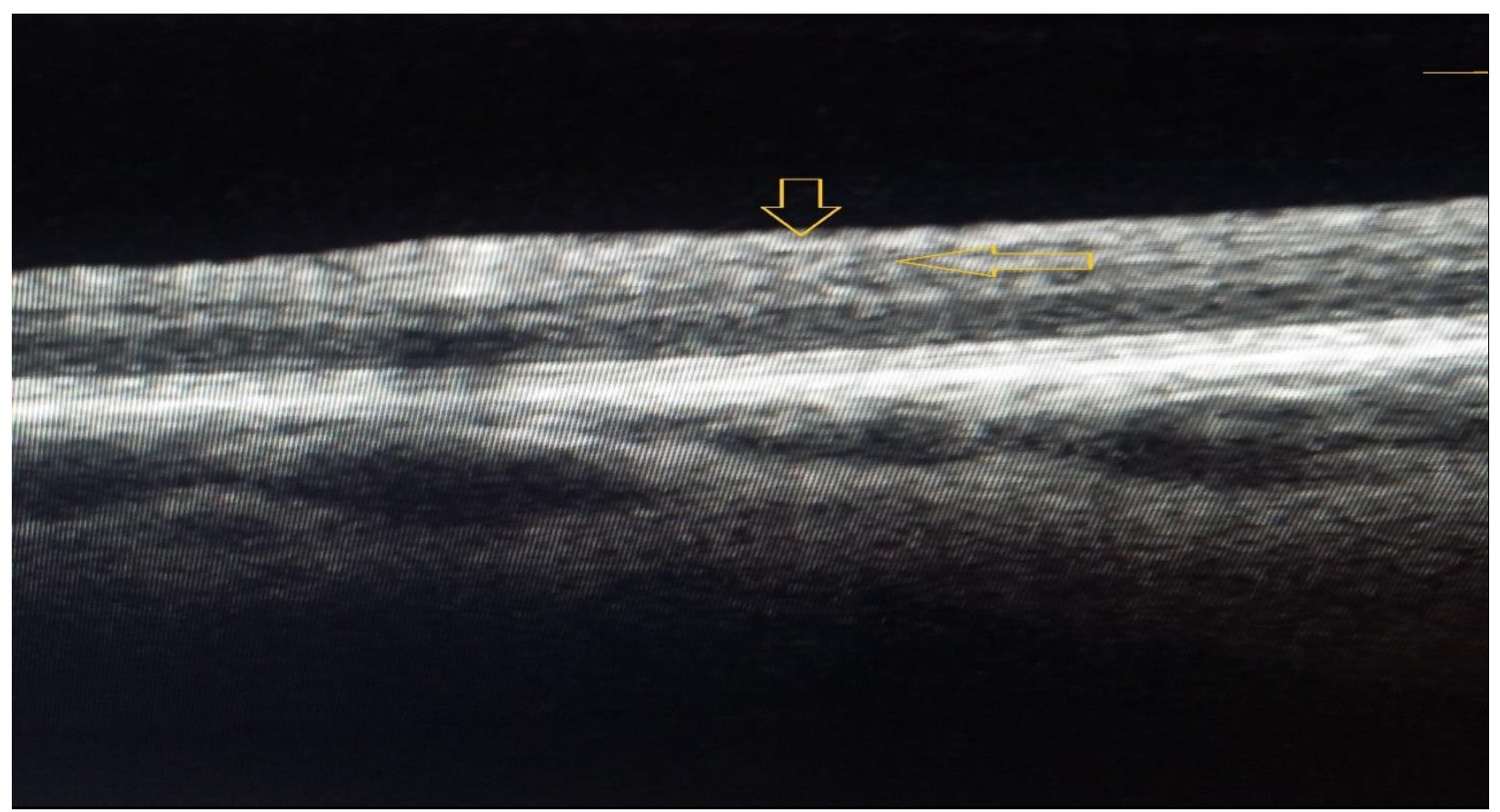

Figure 4: Tau tangle. 


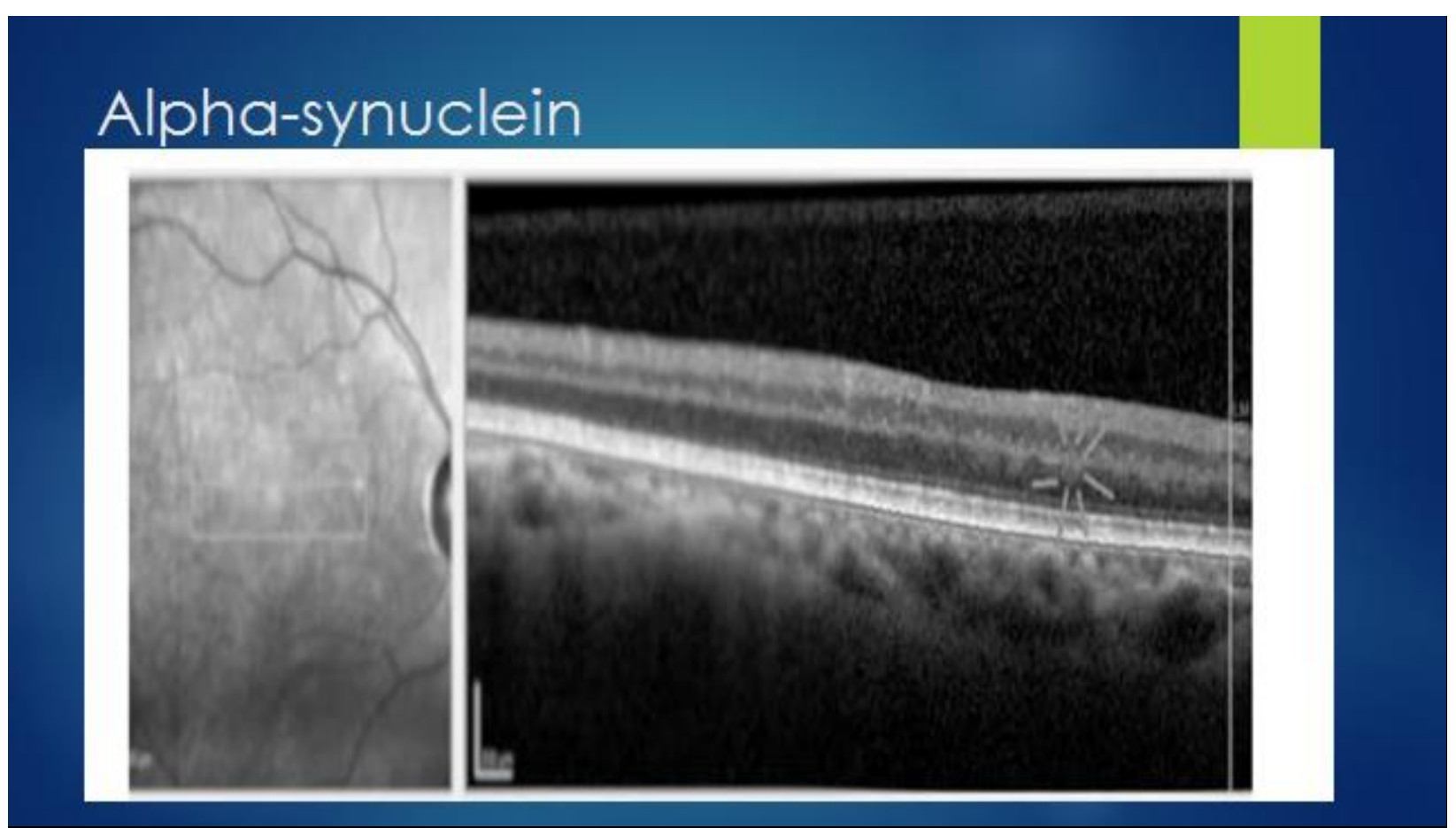

Figure 5: Alpha-synuclein.

\section{Discussion}

Although accumulation of alpha-synuclein has mostly been reported in Parkinson's disease, it is Also a hallmark of many neurodegenerative diseases including AD Alpha-synuclein and Tau share common feautures and may work together in harmony. Both of them can form toxic oligomers intracellular aggregates and evidence suggests that interactions between them spread neurodegeneration. Studies showed that phospho-tau and alpha-synuclein were found together in neurofibrillary tangles, Lewy bodies and neurites. $(13,14)$.

A major difference between Tau and alpha-synuclein is that alpha-synuclein has the capability to Self- aggregate, whereas Tau cannot aggregate by itself and requires an inducing agent. It was demonstrated that alpha-synuclein and Tau promote each other's aggregation in Vitro These observations demonstrated that tau accelerated alpha-synuclein polymerization and that alphasynuclein could act as an inducing agent of tau aggregation (15).

There has been a long- recognized association between repeated mild brain trauma and cognitive Decline Extensive microglial activation leading to neuroinflammation and degeneration has been Suggested as the cause of chronic encephalopathy TBI can cause rapid elevations in beta amyloid plaques and Tau phosphorylation. (3)

Neuroradiological changes are consistent with volume loss, white matter changes and hippocampal atrophy. (16)

Since retina is the extension of the brain, accumulations in different layers directly reflect the neuropathological changes. In our study, beta amyloid plaques in outer plexiform layer which is 
the synaptic region having similarities with the hippocampal neurons may be the identification of the TBI pathology in the brain.Tau phosphorylation is implied as the main pathology that progresses the disease and beta- amyloid accumulation is influenced by it. Plus, in animal studies immunotherapy that targets tau protein can slow the cognitive decline. (17) Monitoring tau has been extremely important according to recent research, and retina is the most easily accessed region that can offer this opportunity.Our study confirms the parallel findings in retina in AD and TBI. This is the first study that identified retinal plaque and Tau accumulation in TBI.

\section{Conclusion}

TBI may cause retinal neurodegeneration similar to AD.

\section{References}

[1] Prince, M. And Bryce, R. And Ferri, C.: World Alzheimer Report 2011,. Alzheimer's Disease International, 2011

[2] Hyman Bt, Phelps, Ch, Beach Tg, Bigio Eh, Cairns $\mathrm{Nj}$, Carrillo Mc, Et Al. National Institute on Aging- Alzheimer's: Association Guidelines on Neuropathology Assessment of Alzheimer'sdisease. Alzheimers Dement 2012:1-13.

[3] Faden Ai, Loane Dj: Chronic Neurodegeneration after Traumatic Brain Injury. Neurotherapeutics. 12(1); 2015

[4] Mckee Ac, Robinson Me. Military-Related: Traumatic Brain Injury and Neurodegeneration. Alzheimers Dement. 2014, 10:242-53

[5] Barnes And Yaffe, K.: The Projected Effect of Risk Factor Reduction on Alzheimer's Disease Prevalence, the Lancet Neurology 10:819-828. 2011.

[6] Blennow, K. And Hampel, H.: Csf Markers For Incipient Alzheimer's Disease,. The Lancet Neurology 2:605-613. 2003

[7] Terman A, Brunk Ut: Lipofuscin. Int J Biochem Cell Biol. 2004 Aug, 36:1400-4.

[8] Seehafer Ss, Pearce Da. You Say Lipofuscin: We Say Ceroid: Defining Autofluorescent Storage Material Apr. 2006, 27:576-88.

[9] Nandakumar N, Buzney S, Weiter Jj. Lipofuscin: And the Principles of Fundus Autofluorescence: A Review. Semin Ophthalmol. 2012 Sep-Nov, 27:197-201.

[10] Maya Koronyo-Hamaoui, S. Yosef Koronyo, Alexander V. Ljubimov, Carol A. Miller, Minhee K. Ko, Keith L. Black Et Al. Identification: Amyloid Plaques in Retinas From Alzheimer's Patients and Noninvasive In Vivo Optical Imaging Of Retinal Plaques In A Mouse Model. Neuroimage. 2011 January, 54:204. S217

[11] Bloudek Lm, Spackman Ed, Blankenburg M: Sullivan Sd.Review And Meta-Analysis Of Biomarkers And 55 Diagnostic Imaging In Alzheimer's Disease. J Alzheimers. Dis, 2011:627-45.

[12] Clinic, Mayo: Tau A Driver of Alzheimer's Disease, Study of Thousands of Brains Reveals. Sciencedaily. March 2015,

[13] Ishizawa T, Mattila P, Davies P, Wang D, Dickson: Dw. Colocalization of Tau and Alphasynuclein Epitopes in Lewy Bodies. J Neuropathol Exp Neurol. 2003, 62:389-397.

[14] Simon Moussaud, Daryl R Jones, Elisabeth L: Moussaud-Lamodière. Alpha-Synuclein And Tau: Teammates In Neurodegeneration?. Molecular Neurodegeneration. 2014, 9:43

[15] Badiola N, De Oliveira Rm, Herrera F, Guardia-Laguarta C, Goncalves Sa, Pera M:

[16] Suarez-Calvet M, Clarimon J, Outeiro Tf, Leo A: Tau Enhances Alpha-Synuclein Aggregation And Toxicity In Cellular Models Of Synucleinopathy.. Plos One. 2011, 6:26609. 
[17] Shenton Me, Hamoda Hm, Schneiderman Js: Et Al.. A Review Of Magnetic Resonance Imaging And Diffusion Tensor Imaging Findings In Mild Traumatic Brain Injury. Brain Imaging Behav 2012:137-192.

[18] Casstillo-Carranza Dl, Guerrero-Muñozmj: Sengupta U. Tau Immunotherapy Modulates Both Pathological Tau And Upstream Amyloid Pathology In An Alzheimer's Disease Mouse Model.The. Journal of Neuroscience 2015, 35:4857-4868

\footnotetext{
*Corresponding author.

E-mail address: kayabasi@yahoo.com
} 